\title{
Fleeting images: A new look at early emotion discrimination
}

\author{
MARKUS JUNGHÖFER, ${ }^{\mathrm{a}, \mathrm{b}}$ MARGARET M. BRADLEY, ${ }^{\mathrm{a}}$ THOMAS R. ELBERT, ${ }^{\mathrm{b}}$ \\ AND PETER J. LANG ${ }^{\mathrm{a}}$ \\ ${ }^{a}$ University of Florida, Gainesville, USA \\ ${ }^{\mathrm{b}}$ University of Konstanz, Konstanz, Germany
}

\begin{abstract}
The visual brain quickly sorted stimuli for emotional impact despite high-speed presentation ( 3 or 5 per s) in a sustained, serial torrent of 700 complex pictures. Event-related potentials, recorded with a dense electrode array, showed selective discrimination of emotionally arousing stimuli from less affective content. Primary sources of this activation were over the occipital cortices, extending to right parietal cortex, suggesting a processing focus in the posterior visual system. Emotion discrimination was independent of formal pictorial properties (color, brightness, spatial frequency, and complexity). The data support the hypothesis of a very short-term conceptual memory store (M. C. Potter, 1999)—shown here to include a fleeting but reliable assessment of affective meaning.
\end{abstract}

Descriptors: Emotion, ERP, RSVP, Pictures, Attention capture

This research introduces a new method in emotion studies-rapid serial visual presentation (RSVP; see Potter, 1999) — in an experiment that assesses early affective discrimination in the visual brain. In this paradigm, the participant is exposed to a sustained flood of visual stimuli, at rates that can exceed the average fixation period of the eye. Based on cognitive research using this rapid presentation technique, Potter proposes that the brain has a first, very short-term conceptual memory system, in which stimuli reach meaningful representation rapidly, but consolidation is transient. Thus, when exposed to a rapid stream of pictures, participants can detect particular content, based on prior instruction, but memory for individual stimuli is little better than chance. As Intraub (1999) notes, "the subjective experience is ... one of grasping and losing large amounts of information within moments" (p. 66). Eventrelated potentials (ERPs) are assessed here to determine if this "attention capture" (Gibson \& Kelsey, 1998) and very early conceptual analysis includes emotion discrimination, and if the affective processing is localized in the visual cortex.

Recent research emphasizes that motivational/emotional relevance is a primary determinant of selective attention: Somatic, autonomic, and cortical events associated with orienting are automatically activated by emotionally arousing representations in a

This research was supported by grants from the National Institute of Mental Health (MH37757, MH43975, MH52384), the Deutsche Forschungsgemeinschaft, and the Institut für Grenzgebiete der Psychologie und Psychohygiene. Thanks to Sabine Harbecke, who assisted in data reduction.

Address reprint requests to: Markus Junghöfer, Box 100165, Health Sciences Center, University of Florida, Gainesville, FL 32610-0165, USA. E-mail: junghofe@ufl.edu. variety of paradigms, independent of instructional direction (Lang, Bradley, \& Cuthbert, 1997). Brain imaging research shows, furthermore, that this natural selective attention to emotional picture content is accompanied by widespread activation of occipital cortex-suggesting that the visual system is specially tuned to detect and process motivationally relevant stimuli (Lang et al., 1998). These PET and fMRI studies depend, however, on slow changes in blood flow, and are insensitive to early stages in the perceptual process. Although ERP research (e.g., Cuthbert, Schupp, Bradley, Birbaumer, \& Lang, 2000) suggests that affect discrimination can have early onset, most studies used relatively long presentation periods, and modest electrode arrays that do not permit the high topographical resolution needed to locate underlying generators (i.e., in visual system). It is as yet undetermined if affective differences can be captured when exposure is brief and the processing load is high-with affective quality changing in milliseconds, at the speed of the scanning eye.

ERPs were recorded here (International Affective Picture System [IAPS] Center for the Study of Emotion and Attention, 1999) from a 129-electrode array. Each participant viewed 700 pictures, varying in affective arousal, briefly presented in a continuous input flood $(3$ or $5 \mathrm{~Hz}$ ) - speeds that compromise recognition memory even in a short stimulus series (Potter, 1999). The research was designed to determine if the brain discriminates varying affective stimuli when presented in the rapid serial visual presentation torrent, and furthermore, if the early conceptual discrimination implicated by this method has a visual cortex focus. Control conditions test if ERP differences are related to conceptual processing, independent of formal sensory characteristics, such as color, spatial frequency, and complexity. 


\section{Method}

\section{Participants}

Participants were 12 naive general psychology students at the University of Florida and 5 researchers at the Center for the Study of Emotion and Attention, with no personal or family history of epilepsy. Due to equipment problems, $N$ varied slightly for different videos, resulting in $n=17$ for alternating videos, $n=11$ (naive) for random videos, and $n=13$ (11 naive, 2 lab) for gray-scale videos.

\section{Experimental stimuli and design}

Computerized video movies were made using Adobe ${ }^{\circledR}$ Premiere ${ }^{\circledR}$ software on a G3 Power Macintosh ${ }^{\circledR}$. Each video used all 704 pictures in the 1999 IAPS, presented in a serial stream (30 frames per s), synchronized to the video beam sweep, without perceivable interstimulus intervals $(60 \mathrm{~Hz}$ refresh rate). Each picture was exposed for 10 frames (333 $\mathrm{ms}$ in the "fast" video) — sufficient time for major components of the ERP to develop, or for 6 frames (200 ms in the "very fast" video-approaching the highest rapid presentation rates studied (Potter, 1999), but potentially limiting ERP development. Based on standardized ratings of arousal (Lang, Bradley \& Cuthbert, 1999), the IAPS pictures were divided at the median into pictures high and low in emotional arousal. In the main design, pictures rated high and low in emotional arousal were presented in an alternating sequence (e.g., high-low-high-low, etc.), allowing analysis by stimulus pairs (as there is no true baseline).

Five control videos were presented: (1) The same pictures as above were presented in random order (not alternating) at the fast rate $(3 \mathrm{~Hz})$ or $(2)$ at the very fast rate $(5 \mathrm{~Hz}) ;(3)$ the same pictures were presented in black-and-white (5 Hz, alternating format); (4) videos of checkerboards (not pictures) were presented, alternating in brightness $(3 \mathrm{~Hz})$ or $(5)$ spatial frequency $(3 \mathrm{~Hz})$.

The first four videos presented were the fast and very fast versions of the alternating and randomized IAPS pictures, with order counterbalanced across subjects. The black-and-white video was always presented in the fifth position, followed by the two checkerboard videos (order counterbalanced across subjects).

\section{Procedure and apparatus}

A 129-sensor system (Electrical Geodesic ${ }^{\circledR}$ Inc., Eugene, OR) was used, fitting the electrode net so that a point midway between nasion/inion and left/right mastoids defined the $\mathrm{Cz}$ reference. Scalp impedance of each sensor was below $50 \mathrm{k} \Omega$. EEG data $(0.1-50 \mathrm{~Hz})$ were continuously recorded, at a sampling rate of $125 \mathrm{~Hz}$. Videos were projected via LCD projector from an adjacent room onto a screen (width $95 \mathrm{~cm}$ ), approximately $200 \mathrm{~cm}$ in front of the participant.

The subject room was darkened. Participants were instructed to maintain focus on the screen's center, marked by a red laser dot. Presentation time was approximately $4 \mathrm{~min}$ for the each of the 4 "fast" videos $(333 \mathrm{~ms})$ and $2.5 \mathrm{~min}$ for each of the 3 "very fast" (200 ms) videos.

Visual ERPs were artifact corrected and averaged as recommended by Junghöfer, Elbert, Tucker, \& Rockstroh (2000).

\section{Results}

Fast (3 Hz= $333 \mathrm{~ms})$ presentation

Figure 1 (upper left panel) shows the grand averages for visual ERPs, evoked by pictures high and low in emotional arousal, at a characteristic right occipital electrode (EGI array, \#85). Three wave-

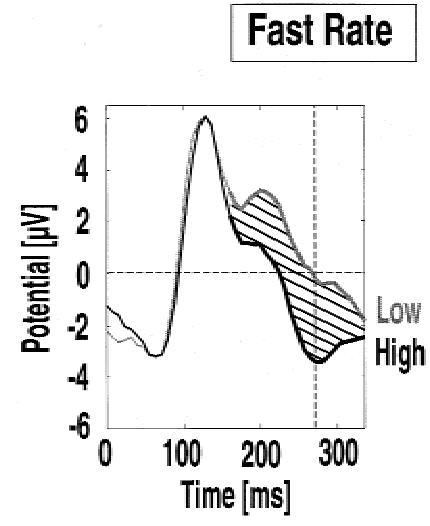

(3 Hz; $333 \mathrm{~ms}$ )
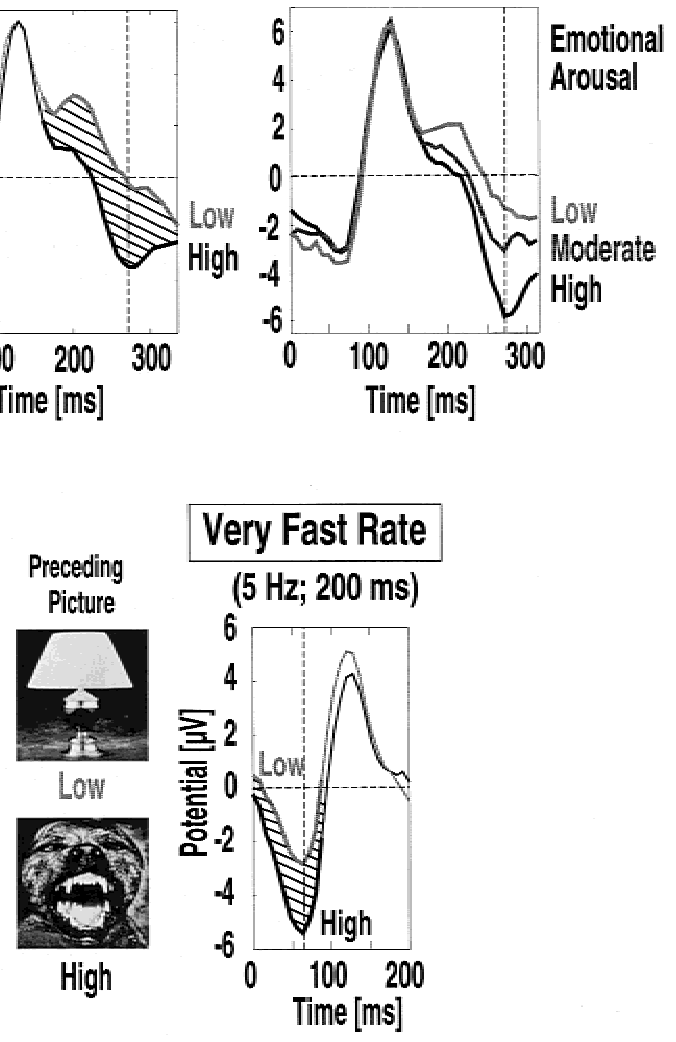

Figure 1. Upper left: Visual event-related potentials (ERPs) for pictures presented at the fast $(3 \mathrm{~Hz})$ rate at a right occipital recording site when viewing pictures judged high or low in emotional arousal. Upper right: ERPs at the same recording site when viewing pictures judged low, moderate, or high in emotional arousal. Lower panel: ERPs for pictures presented at the very fast $(5 \mathrm{~Hz})$ rate as a function of the emotional arousal of the preceding picture.

form components are apparent: P100 (peak at $128 \mathrm{~ms})$, P200 (200 ms peak), and N260 (264 ms peak), and were analyzed for each hemisphere, averaged across all posterior electrodes (26 sites per hemisphere), based on mean potentials over intervals 96-160 ms (P100), 168-232 ms (P200), and 232-296 ms (N260). A mixed model analysis of variance (ANOVA), with a between-subject factor of prior experience (2: naive or lab member), and three repeated measures of Emotional Arousal (2: low or high), Time (3: P100, P200, or N260), and Hemisphere (2: right or left) was conducted on the mean potentials. There were no effects or interactions involving prior experience viewing the IAPS in these analyses.

For the alternating video, visual ERPs differed according to emotional arousal over time: Emotional arousal, $F(1,15)=25.2$, $p<.001$, time $F(2,30)=14.54, p<.001$, and their interaction, $F(2,30)=29.6, p<.001$. No difference as a function of picture content was found at the early P100 component, $F(1,15)<1$. However, the high arousal waveform became progressively more negative than the low arousal ERP, beginning around $150 \mathrm{~ms}$ (Figure 1), and differed significantly for both the P200, $F(1,15)=$ $19.3, p<.001$ and the N260 component, $F(1,15)=112.1, p<$ .001 . Moreover, this difference was larger at the later, N260 component than for the P200 component, $F(1,15)=12.1, p<.001$. 
For the N260 component, all 17 subjects showed this difference. Although emotional arousal similarly affected ERPs in both the right, $F(1,15)=30.0, p<.001$, and the left, $F(1,15)=18.6, p<$ .001 , hemispheres, the effect was larger on the right hemisphere, arousal $\times$ hemisphere interaction, $F(1,15)=10.2, p<.006$.

As a further test of the sensitivity of emotional arousal discrimination, ERPs in the right hemisphere for the alternating video were averaged across scarcely, moderately, and highly arousing pictures ${ }^{1}$ (see Figure 1, upper right panel): Emotionally arousing pictures prompted a larger N260 than did moderately arousing stimuli, $t(10)=7.94, p<.001$, and moderately arousing pictures prompted more negativity then did scarcely arousing pictures, $t(10)=3.38$, $p<.001$.

\section{Very fast $(5 \mathrm{~Hz}=200 \mathrm{~ms})$ presentation}

Inspection of sequential ERPs suggested that the N60 component reflected continued processing of the preceding picture. Accordingly, the mean potential centering on this peak was computed as described above. Figure 1 (bottom) illustrates ERPs in the very fast presentation condition when the preceding picture was high or low in emotional arousal.

Consistent with the hypothesis that the N60 reflects the same effects found for the N260 component, pictures high in emotional arousal were associated with a more negative potential than low arousal pictures, $F(1,15)=31.9, p<.001$, and this effect was again significant for both left, $F(1,15)=17.8, p<.001$, and right hemispheres, $F(1,15)=41.0, p<.001$, but with a stronger effect in the right hemisphere, arousal by hemisphere interaction, $F(1,15)=$ $11.1, p<.001$.

\section{Topography}

Figure 2 illustrates the spherical spline-based Laplacian maps (Junghöfer, Elbert, Leider, Berg, \& Rockstroh, 1997), proportional to the negative CSD, computed for N260 (fast) and N60 (very fast) components, using the difference in potentials between pictures high and low in emotional arousal. The strongest activations were clearly located in the occipital-parietal area (top panel), relative to frontal or central areas. The high resolution Laplacian maps (bottom panel of Figure 2) show a separation of hemispheric occipitoparietal generators. As for amplitudes, the scalp distribution for the N260 and N60 components are strikingly similar: The underlying sources for the N260 (fast video) and the N60 (very fast video) generated almost identical bilateral and symmetric occipital sinks and sources, as well as twin right hemispheric parietal sink sources.

\section{Color, Brightness, Spatial Frequency}

ERP differences for emotional pictures were not limited to videos in which high and low arousal regularly alternated. When pictures were presented randomly, emotionally arousing pictures again showed a larger N260 component than pictures lower in arousal, both at the fast rate, $t(10)=t=5.3, p<.001$, and the very fast rate, $t(10)=4.6, p<.001$. Visual ERP differences were not an artifact of color, as a larger N60 for emotionally arousing pictures was also obtained for gray-scale pictures, $t(12)=5.2, p<.001$. Analysis of ERPs to checkerboard videos that varied in either brightness or spatial frequency elicited — as expected-bilateral occipital differences. However, the time course, amplitude, scalp and

${ }^{1}$ Pictures were categorized by normative IAPS arousal ratings (1-9 scale; Lang et al., 1999). Low arousal pictures, <4.75; moderate pictures, $>4.7$ and $\leq 5.7$; high arousal pictures, $>5.7$.

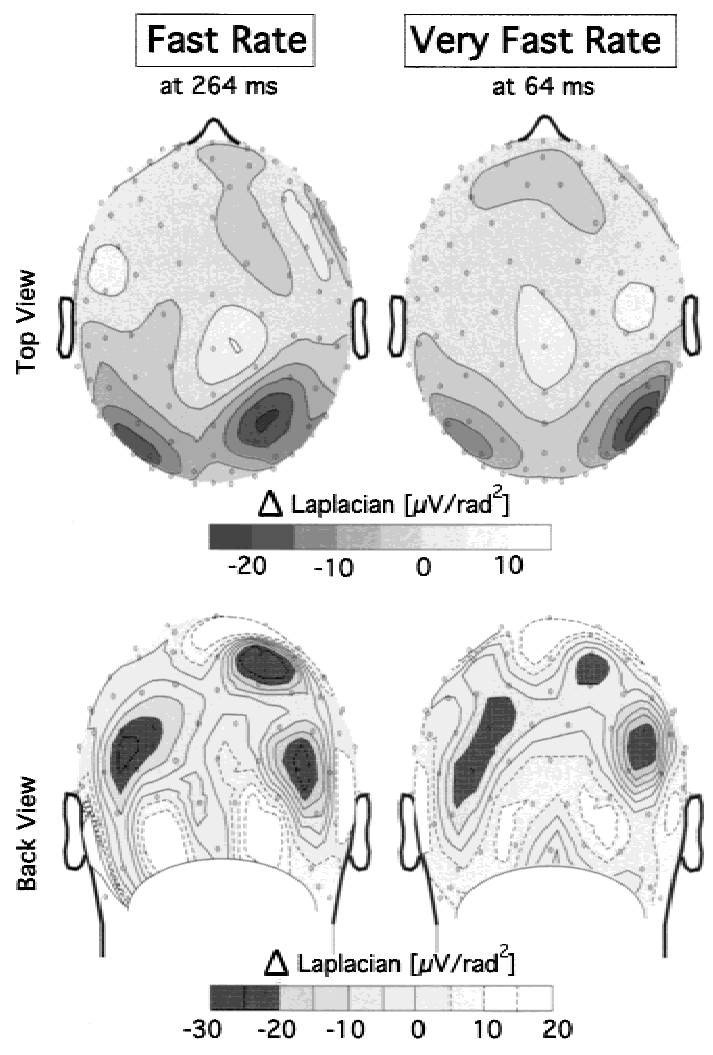

Figure 2. Top: Topographical current source density distributions (Laplacian), viewed from the top of the head, computed on the difference between pictures judged high and low in arousal at N260 for the fast rate (left) and at N60 for the very fast rate (right). The analysis shows that the greatest activity was at posterior sites for both input rates. Bottom: High-resolution current source density topography of the occipital-parietal cortex, showing the emotional arousal difference at N260 for the fast (left) and very fast input rate (right). The fast and very fast stimulus input rates show very similar amplitude and scalp distribution.

Laplacian distributions and asymmetries of these differences were clearly different from those observed for emotional arousal.

\section{Perceptual Complexity}

Several measures of complexity were computed to explore the possibility that this formal variable might mediate emotional arousal effects: (1) JPEG file size (4 variables; simple pictures are highly compressible), (2) singular value decomposition (25 variables; simple pictures can be described with fewer dominant singular values), and (3) spatial frequency distribution (66 variables; e.g., simple pictures show a lower ratio of high to low spatial frequencies). For the fast, alternating video, separate hierarchical regression analyses were conducted on the N260 component, using picture as the random factor, and with complexity measures entered before the arousal variable. In each case, a significant difference in response to pictures high and low in arousal was obtained, $F(1,698)=89.4$, $p<.001$ for JPEG size; $F(1,677)=94.9, p<.01$ for singular value; $F(1,638)=83.1, p<.001$ for spatial frequency.

\section{Discussion}

The findings show rapid, selective "attention capture" in a sustained high-speed input stream of complex picture stimuli. ERPs 
were more negative for high than low arousal pictures, beginning around $150 \mathrm{~ms}$ after picture onset (see Figure 1), with a maximum difference at approximately $260 \mathrm{~ms}$. This was seen clearly even at the fastest rate $(200 \mathrm{~ms} /$ picture), where the ERP waveform difference persisted $60 \mathrm{~ms}$ after stimulus offset-in the perceptual presence of the next stimulus. The data support the view that emotion discrimination occurs at a first stage of conceptual stimulus processing (see Potter, 1999).

Early ERP discrimination of emotional arousal was reliable across the paradigms studied, occurring in both the random picture and alternating high-low arousal conditions. Control analyses provide strong evidence that affect discrimination of IAPS pictures is independent of formal visual properties of the stimuli, including color, brightness, spatial frequency, and complexity.

The main neural sources for early emotion discrimination appear to be located in primary and secondary visual processing areas of the brain. Source analyses of emotional arousal differences at both presentation rates generated near identical scalp distributions-bilateral over the occipital cortex, with an adjacent source over right parietal cortex. Determining whether the larger right-side potentials reflect asymmetric activation of parietal generators only (as Figure 2 suggests) or if occipital sources also show asymmetric effects will require further research. The topography broadly mirrors previous fMRI research, which also found more extensive activity for emotional pictures over occipital and parietal sites, with a right hemisphere emphasis (Lang et al., 1998).

While ERP emotion discrimination is clear, it is not obvious that such high-speed serial presentation of varied pictures actually evokes affective states. The differential ERP may represent only an initial conceptual processing that is limited to the sensory system. Of course, 150-200 ms is more than enough time for re-entrant projections from cingulate or amygdala to participate in affect analysis; however, it may be insufficient for general response mobilization in either defense or appetite. From a cognitive perspective, Potter (1999) questions whether participants are wholly conscious of individual pictures when viewed at high presentation rates, proposing that first stage selection and conceptual structuring may happen before one becomes aware. Interestingly, in the current experiment, no participant could report which of the justviewed videos presented stimuli randomly, and which systematically alternated high and low arousal pictures.

Harnessing ERP analysis to the RSVP methodology provides a new look at early emotion processing and opens several promising avenues for research. It will be important to relate these ERP effects to picture memory and affective awareness, using the procedures already developed by Potter and Intraub (1999). Unlike the iconic masking used in recent emotion research (e.g., rearranged shards of the target stimulus), this rapid presentation procedure involves conceptual masking, as each stimulus is followed by a new semantically valid replacement. The two methods involve different timing, and appear to interrupt different stages of processing, with potentially unique effects on emotional memory.

Research now underway will assess possible differences in ERP discrimination in the rapid processing of pleasantly and aversively arousing stimuli. It is also planned to test the speed limit of ERP affect discrimination, which already shows a spill-over effect (parallel processing?) at the $200 \mathrm{~ms}$ per picture input rate. Finally, the method commends itself for use in clinical assessment, where many stimuli (clinically relevant and control) can be presented in a brief period, while affect discrimination is evaluated. Importantly for patients, assessment may occur without full activation of a distressing affective response.

In summary, this research found that ERPs can systematically differentiate emotional from more mundane input, selectively, within a serial flood of discrete visual events. Rapid detection and perceptual processing of emotionally salient stimuli-in a single scan, as stimuli occur fleetingly, or as the eye flicks rapidly through a scene-makes good evolutionary sense. The new methodology described above highlights a motivational imperative: In the natural environment, quick discrimination of cues to appetite or threat provides a survival advantage.

\section{REFERENCES}

Center for the Study of Emotion and Attention [CSEA-NIMH] (1999). The international affective picture system: Digitized photographs. Gainesville, FL: The Center for Research in Psychophysiology, University of Florida.

Cuthbert, B. N., Schupp, H. T., Bradley, M. M., Birbaumer, N., \& Lang, P. J. (2000). Brain potentials in affective picture processing: Covariation with autonomic arousal and affective report. Biological Psychology, 52, 95-111.

Gibson, B. S., \& Kelsey, E. M. (1998). Stimulus-driven attentional capture is contingent on attentional set for displaywide visual features. Journal of Experimental Psychology: Human Perception and Performance, 24, 699-706.

Intraub, H. (1999). Understanding and remembering briefly glimpsed pictures: Implications for visual scanning and memory. In V. Coltheart (Ed.). Fleeting memories: Cognition of brief visual stimuli (pp. 47-70). Cambridge, MA: The MIT Press.

Junghöfer, M., Elbert, T., Leiderer, P., Berg, P., Rockstroh, B. (1997). Mapping EEG-potentials on the surface of the brain: A strategy for uncovering cortical sources. Brain Topography, 9, 203-217.

Junghöfer, M., Elbert, T., Tucker, D., Rockstroh, B. (2000). Statistical control of artifacts in dense array EEG/MEG studies, Psychophysiology, 37, 523-532.
Lang, P. J., Bradley, M. M., \& Cuthbert, B. N. (1997). Motivated attention: Affect, activation and action. In P. J. Lang, R. F. Simons, \& M. T. Balaban (Eds.). Attention and orienting: Sensory and motivational processes. Hillsdale, NJ: Erlbaum.

Lang, P. J., Bradley, M. M., \& Cuthbert, B. N. (1999). International affective picture system (IAPS): Instruction manual and affective ratings. Technical Report A-4. Gainesville, FL: The Center for Research in Psychophysiology, University of Florida.

Lang, P. J., Bradley, M. M., Fitzsimmons, J. R., Cuthbert, B. N., Scott, J. D., Moulder, B. \& Nangia, V. (1998). Emotional arousal and activation of the visual cortex: An fMRI analysis. Psychophysiology, 35, 199210

Potter, M. C. (1999). Understanding sentences and scenes: The role of conceptual short-term memory. In V. Coltheart (Ed.), Fleeting memories: Cognition of brief visual stimuli (pp. 13-46). Cambridge, MA: The MIT Press. 